\title{
Impact of Doping a Phytosteryl Sulfate on the Properties of Liposomes Made of Saturated and Unsaturated Phosphatidylcholines
}

\author{
Risa Tanaka ${ }^{1}$, Ananda Kafle ${ }^{1 *}$, Masaaki Akamatsu', Avinash Bhadani ${ }^{2}$, Kenichi Sakai ${ }^{1,2}$, \\ Chihiro Kaise ${ }^{2,3}$, Teruhisa Kaneko ${ }^{2,3}$, and Hideki Sakai ${ }^{1,2 *}$ \\ ${ }^{1}$ Department of Pure and Applied Chemistry, Faculty of Science and Technology, Tokyo University of Science, 2641-Yamazaki, Noda, Chiba, \\ 278-8510, JAPAN \\ ${ }^{2}$ Research Institute for Science and Technology, Tokyo University of Science, 2641-Yamazaki, Noda, Chiba, 278-8510, JAPAN \\ ${ }^{3}$ L. V. M. C. Inc. Komagome-7-14-3, Toshima-ku, Tokyo, 170-0003, JAPAN
}

\begin{abstract}
The size, dispersibility, and fluidity of DPPC (1,2-dipalmitoyl-sn-glycero-3-phosphocholine), POPC (1-palmitoy-2-oleoyl-sn-glycero-3-phosphocholine), and DOPC (1,2-dioleoyl-sn-glycero-3phosphocholine) liposomes doped with $\beta$-sitosteryl sulfate $\left(\mathrm{PSO}_{4}\right)$ were comparatively studied. In all three types of liposomes, $\mathrm{PSO}_{4}$ reduced sizes and enhanced the negative values of the $\zeta$-potential. However, the effect on sizes quantitatively differed in the three cases in a manner dependent on their phase behaviors. $\mathrm{PSO}_{4}$ rigidified each type of membrane in its liquid crystalline phase and fluidized the gel phase. It enhanced the glucose trapping efficiency (TE) of both DPPC and DOPC liposomes. The TE of DPPC first increased with the increasing concentration of $\mathrm{PSO}_{4}$, then decreased gradually. On the other hand, in the case of DOPC, the TE increased significantly upon addition of $\mathrm{PSO}_{4}$, then remained nearly constant. Though the exact dependence of $\mathrm{TE}$ on the $\mathrm{PSO}_{4}$ concentration differed in the two cases, its effect, in each case, was more than the effect of $\beta$-sitosterol (POH). The ability of $\mathrm{PSO}_{4}$ for reducing the size and enhancing dispersibility and TE of liposomes can be useful for preparing cosmetics and pharmaceutical formulations.
\end{abstract}

Key words: trapping efficiency, $\beta$-sitosteryl sulfate, phosphatidylcholine, liposome, DPPC, DOPC, POPC

\section{Introduction}

Phospholipids (Fig. 1) are amphiphilic molecules which possess a hydrophilic headgroup and a lipophilic tail consisting of one or two acyl chains. When mixed with water, the lipid molecules undergo self-assembly forming lamellar bilayers. These bilayers form the structural backbone of biological membranes, and therefore, are studied as models for understanding the membrane phenomenon ${ }^{1-3)}$. At low temperatures, they exist in a significantly rigid structure known as gel phase. At temperatures above a threshold called main phase transition temperature $\left(\mathrm{T}_{\mathrm{m}}\right)$, they exist in the fluid, liquid crystalline phase. In dilute dispersions, these membranes take the form of spherical vesicles known as liposomes (Fig. 1). Liposomes are widely used as drug carriers in advanced drug delivery systems (DDS $)^{4,5)}$. Liposomes consist of one or more concentric lipid bilayers that enclose water at the center as well as between successive bilayers $^{6,7)}$. Water-soluble drugs are dissolved in the aqueous core of liposomes and transported into the body. Similarly, lipophilic drugs are loaded in liposome's hydrophobic tail region. Biocompatibility of phospholipids makes them a popular choice for using in DDS. They are also used in skincare cosmetics ${ }^{8,9}$.

Steroids constitute a class of organic compounds possessing a characteristic arrangement of four fused ring systems. The members of this family having an - $\mathrm{OH}$ functional group are called sterols. Among numerous steroids found in organisms, cholesterol in animals and phytosterols in plants serve as important constituents in biomembranes $^{10,11}$. Cholesterol sulfate is also present in various animal tissues ${ }^{12,13)}$. The major function of sterols in the membrane is to optimize its fluidity and permeability. By locating themselves between phospholipid molecules in the membrane, sterols can fluidize the membrane if it is initially in a gel phase and rigidify if it is in the liquid crystalline phase $^{14-16)}$. In liposomes too, sterols are used to achieve

\footnotetext{
*Correspondence to: Ananda Kafle, Department of Pure and Applied Chemistry, Faculty of Science and Technology, Tokyo University of Science, 2641-Yamazaki, Noda, Chiba, 278-8510, JAPAN; Hideki Sakai, Department of Pure and Applied Chemistry, Faculty of Science and Technology, Tokyo University of Science, 2641-Yamazaki, Noda, Chiba, 278-8510, JAPAN.

E-mail: hisakai@rs.tus.ac.jp (HS); anandakafle@ @r.tus.ac.jp (AK) ORCID ID: Kenichi Sakai (https://orcid.org/0000-0003-40388954)
} 


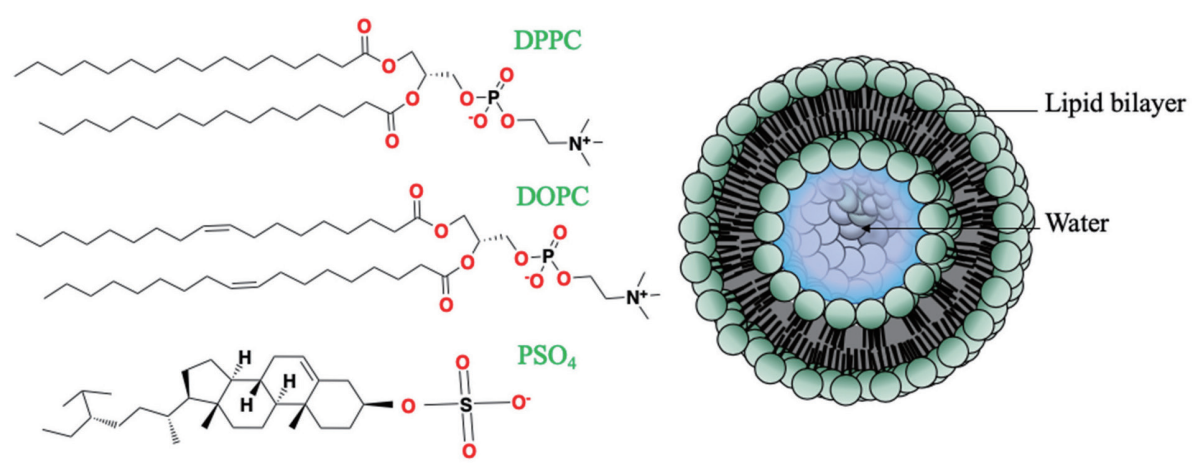

Fig. 1 Left- DPPC, DOPC and $\beta$-sitosteryl sulfate $\left(\mathrm{PSO}_{4}\right)$ molecules. Right- A liposomal particle formed from lipid assembly.

the same function as in natural membranes. Optimizing the fluidity of liposomal particles ensures their proper transport in the body, efficient retention of the drug and its timely release in the intended tissue ${ }^{9,17)}$. Conventionally, cholesterol is being used for this purpose in cosmetic and pharmaceutical formulations. However, recently, cholesterol is found to transmit a fatal mammalian disease known as prion disease ${ }^{3,18)}$. Therefore, an alternative to this sterol is necessary.

Previously, we carried out studies on the systems consisting of phospholipids and plant steroids ${ }^{3,9,19,20)}$. We found that $\beta$-sitosteryl sulfate, a derivative of $\beta$-sitosterol, efficiently fluidizes and enhances hydration of the membranes made of saturated phosphatidylcholines (PCs). It also improves dispersibility and stability of the liposomal particles against agglutination ${ }^{9)}$. These properties of $\beta$-sitosteryl sulfate are valuable for the preparation of chemically and physically stable liposomes from saturated phosphatidylcholines. Further, being a plant derived steroid, it is more health-friendlier than cholesterol and is safer towards diseases such as prion disease. Though in our previous studies we shed light on some important aspects of the roles of $\mathrm{PSO}_{4}$ in membranes made of saturated PCs, no study has yet been carried out in another important property, the trapping efficiency (TE) of such liposomes, which is instrumental for effective performance of a drug carrier. Further, though saturated phospholipids are better for preparing chemically stable liposomes, phospholipids with unsaturated hydrocarbon chains are also preferred frequently for their higher fluidity and flexibility. Despite this, no study has so far been carried out on the effects of $\mathrm{PSO}_{4}$ on the properties of the unsaturated PCs such as DOPC (1,2-dioleoyl-sn-glycero-3-phosphocholine) and POPC (1-palmitoyl-2-oleoyl-sn-glycero-3-phosphocholine). In this paper, we are reporting a comparative study on the size, dispersibility, fluidity, and trapping efficiency of the DPPC and DOPC liposomes containing $\mathrm{PSO}_{4}$.

\section{Materials and Methods}

\subsection{Materials}

DPPC, DOPC, and POPC (each 99\% pure)were purchased from the NOF Corporation, Japan and were used without further purification. $\mathrm{PSO}_{4}$ and one of the samples of $\beta$-sitosterol (POH) were obtained from LVMC Inc., Tokyo, Japan, and purified as described elsewhere ${ }^{3)}$. Another POH sample was purchased from Sigma. The fluorescence probe 1,6-diphenyl-1,3,5-hexatriene(DPH, >98\% purity) was obtained from Sigma-Aldrich. The coloring reagent used for UV-Vis spectrophotometry was purchased from Wako Pure Chemicals Industries Ltd.

\subsection{Sample preparation}

For the preparation of samples for the measurement of size, fluidity, and $\zeta$-potential, appropriate quantities of DPPC, DOPC or POPC, and $\mathrm{PSO}_{4}$ were weighed to obtain mixtures of varying compositions in which the mole fraction of $\mathrm{PSO}_{4}(x)$ ranged from 0 to 0.5 . The mixtures were then dissolved in a solvent ${ }^{3,21)}$ containing, respectively, 3 and 1 parts by volume of chloroform and methanol. A current of $\mathrm{N}_{2}$ gas was passed into each sample taken in a $60 \mathrm{~mL}$ vial until whole of the solvent was removed. While passing $\mathrm{N}_{2}$, the vial was gently rotated so that a thin, uniform lipid film was deposited along its inner walls. The vial was then placed in a vacuum desiccator to remove any remaining traces of the solvent. Ultrapure water was warmed at $62^{\circ} \mathrm{C}\left(\sim 20^{\circ} \mathrm{C}\right.$ above the $\mathrm{T}_{\mathrm{m}}$ of $\left.\mathrm{DPPC}\right)$ and added to the dry lipid film so as to obtain a suspension with 2 $\mathrm{mmol} / \mathrm{L}^{-1}$ solid concentration. The suspensions were vortex-mixed for about $15 \mathrm{~min}$ at this temperature. The multilamellar vesicles (MLVs) thus obtained were used in further experiments $^{9)}$. For DSC measurement and the measurement of trapping efficiencies (TE), the sample concentration used was $10 \mathrm{mmol} / \mathrm{L}^{-1}$ instead of $2 \mathrm{mmol} / \mathrm{L}^{-1}$. For the measurement of TE, $0.027 \mathrm{M}$ glucose solution was used to hydrate lipids instead of water.

\subsection{Particle size and zeta-potential}

Dynamic light scattering (photon correlation spectroscopy) technique was used to measure particle size distribu- 
tion. A plastic cuvette containing about $1 \mathrm{~mL}$ of the suspension was placed in the sample holder of a Litesizer 500 instrument (Anton Paar, Graz, Austria). Measurements were carried out at $25^{\circ} \mathrm{C}$ with laser light of wavelength 658 $\mathrm{nm}$ produced by a single-frequency laser diode, providing $40 \mathrm{~mW}$. Detection angles of $15^{\circ}, 90^{\circ}$, or $175^{\circ}$, were selected depending on the transmittance of the sample. Fluctuations in the scattered light intensity caused by the moving vesicles in solution were analyzed and the parameters including optical density, size distribution, and mean hydrodynamic diameter were calculated with the help of the Kalliope software. The same instrument was used to measure the $\zeta$-potentials, for which, about $350 \mu \mathrm{L}$ of the sample was filled in an Omega cuvette made from polycarbonate and provided with two gold electrodes. The voltage range of 0.1 $-200 \mathrm{~V}$ for the measurement was set automatically by the instrument ${ }^{9)}$.

\subsection{Fluorescence measurements}

A thin film of 1,6-diphenyl-1,3,5-hexatriene (DPH) dissolved in chloroform was deposited along the inner walls of a glass vial and dried with a current of $\mathrm{N}_{2}$ followed by desiccation. The liposome solution, warmed at $62^{\circ} \mathrm{C}$, was added to the film so that the final DPH concentration was 300 times less than the lipid concentration of the liposome ${ }^{22)}$. The material was vortex mixed and stored in dark overnight at $25^{\circ} \mathrm{C}$. The fluorescence intensities were measured at different temperatures within the range of 25 to $45^{\circ} \mathrm{C}$ using RF-5000 spectrofluorometer (Shimadzu Co., Ltd). The emitted light (wavelength $=450 \mathrm{~nm}$ ) polarized parallel and perpendicular to the excitation radiation (wavelength $=350 \mathrm{~nm}$ ) was used for these measurements. From the obtained fluorescence intensities, the fluorescence anisotropy $(r)$ values were calculated. Fluorescence anisotropy $(r)$ for highly turbid samples were corrected for light scattering effects using a method described elsewhere ${ }^{9,23)}$.

\subsection{Trapping efficiency}

For the evaluation of the glucose-trapping efficiency (glucose-TE or simply TE), liposomes of $10 \mathrm{mM}$ lipid concentration were prepared with $0,0.05,0.075,0.1,0.15,0.2$, $0.3,0.4$ and 0.5 mole fractions $(x)$ of $\mathrm{PSO}_{4}$. For this, the dry lipid film deposited on a vial, as described above, was hydrated with $0.027 \mathrm{M}$ glucose solution followed by vortex mixing at $62^{\circ} \mathrm{C}$. Dialysis technique was used to remove the unentrapped glucose molecules. For dialysis, $1 \mathrm{~mL}$ of the liposome of each composition was filled in a cellulose tube and sealed at both ends with two clips (Fig. S1, Supplementary Information). The tubes were then placed in a $0.014 \mathrm{M} \mathrm{NaCl}$ solution precooled at $\sim 3-5^{\circ} \mathrm{C}$ for 2 hours. The salt solution was stirred with the help of a magnetic stirrer at this temperature for 11 hours. In this duration, the salt solution was changed at intervals of $1 \mathrm{~h}, 1 \mathrm{~h}, 2 \mathrm{~h}, 3$ $\mathrm{h}$ and 4 h. $50 \mu \mathrm{L}$ of liposome solution was taken out from the cellulose tubes and treated with $150 \mu \mathrm{L}$ volume of ethanol. $80 \mu \mathrm{L}$ of the ethanolic solution, which is equivalent to $20 \mu \mathrm{L}$ of the liposome, was taken out into another vial. 3 $\mathrm{mL}$ of an assay containing glucose oxidase (GOD), 4-aminoantipyrine and phenol, was added into this vial, which was warmed for 5 minutes and then shaken for about 30 sec. The absorbances of the glucose-containing samples were measured with the help of a UV-Vis spectrophotometer (Cary 5484, Agilent Technologies, Singapore). From the absorbances obtained, the glucose concentrations were calculated based on the calibration curve. The glucosetrapping efficiency was calculated as follows-

$$
\begin{aligned}
& \text { Trapping efficiency }(\mathrm{TE})(\%) \\
& =\frac{\text { Amount of glucose in liposomes }}{\text { Glucose of the whole system }} \times 100
\end{aligned}
$$

Release of glucose as a function of storage period was also evaluated. Before storage, $\mathrm{N}_{2}$ gas was passed into each vial to avoid any biodegradation of glucose.

\section{Results and Discussion}

\subsection{Sample appearance}

Figure 2 shows the images of the liposome samples made from DPPC, DOPC and POPC with varying amounts of $\mathrm{PSO}_{4}$. Each of the pure PCs forms an opaque, white suspension. Precipitation occurs by the next day in DPPC suspension whereas the other two remain well dispersed for several days. The early precipitation in DPPC sample agrees with our previous study ${ }^{9}$. While the addition of $\mathrm{PSO}_{4}$ reduces the turbidity of each suspension, the effect is highest in DPPC than the other two.

\subsection{Size and $\zeta$-potential}

Figure 3 shows the hydrodynamic diameter $\left(D_{H}\right)$ and $\zeta$-potential of the DPPC and DOPC liposomes as a function of the mole fraction $(x)$ of $\mathrm{PSO}_{4}$. As shown in this figure, the addition of 0.075 mole fraction of $\mathrm{PSO}_{4}$ reduces the $D_{H}$ of the DPPC liposomes by about 4 times. The size further decreases with successive additions of $\mathrm{PSO}_{4}$. This decrease in size upon addition of $\mathrm{PSO}_{4}$ has been attributed to the introduction of negative surface charge in the liposome by $\mathrm{PSO}_{4}$. This negative charge results in an electrostatic repulsion among liposomal particles necessitating an increased vesicle curvature and higher translational entropy ${ }^{24-26)}$. The requirements for the greater vesicle curvature and the higher translational entropy lead to the formation of a larger number of smaller-sized liposomes. A similar behavior was observed in our previous studies on $2 \mathrm{mM}$ and 5 mM DPPC liposomes ${ }^{9}$. The sizes of the DOPC and POPC liposomes are much smaller than that of the DPPC lipo- 


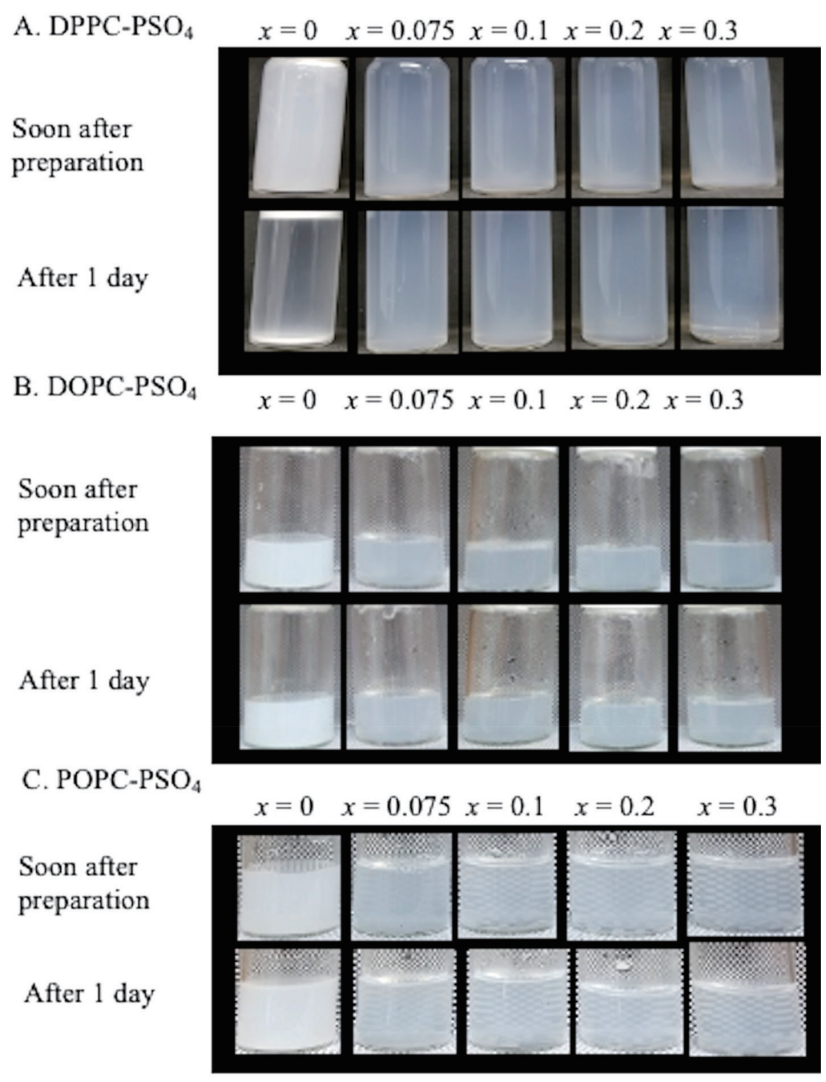

Fig. 2 Appearances of the liposomal suspensions- Top:

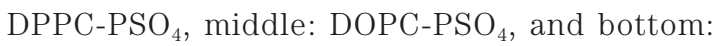
POPC-PSO ${ }_{4}$.

some. This can be attributed to the smaller number of bilayers present in the DOPC and POPC liposome which are in the liquid crystalline $\left(\mathrm{L}_{\alpha}\right)$ phase compared to that in the DPPC which is in a gel $\left(\mathrm{L}_{\beta}{ }^{\prime}\right)$ phase. The increased surface area and decreased shear rigidity in the $\mathrm{L}_{\alpha}$ phase bilayers are responsible for the smaller number of bilayers in this phase ${ }^{27)}$. As the addition of $\mathrm{PSO}_{4}$ leads to a less strong perturbation of the liquid crystalline DOPC and POPC membrane compared to the gel phase DPPC membrane, the

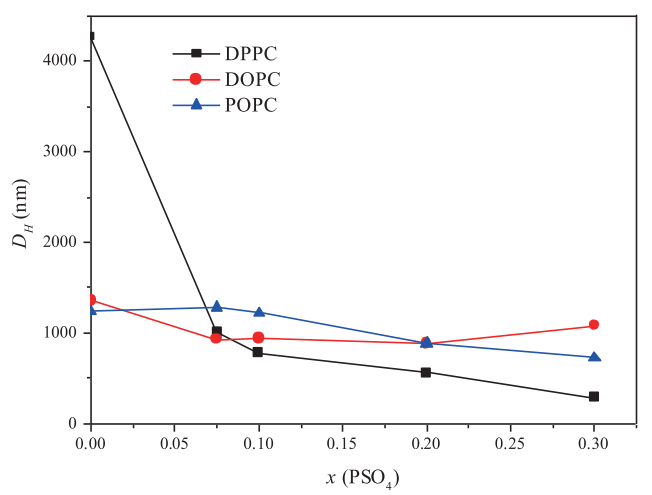

impact of adding $\mathrm{PSO}_{4}$ into these bilayers is accordingly less. For the same reason, the size of the DPPC liposome decreases more rapidly with the addition of $\mathrm{PSO}_{4}$ than the other two.

The negative values of the $\zeta$-potentials for the three pure lipid samples are in the order POPC $>$ DPPC $>$ DOPC, which is in agreement with the previous reports ${ }^{28,29)}$. These increase dramatically with the addition of 0.075 mole fraction of $\mathrm{PSO}_{4}$. This change in the value of $\zeta$-potential is a consequence of the increased negative surface charge of the vesicles owing to the introduction of $\mathrm{PSO}_{4}$ in the membrane. However, instead of decreasing continuously, the $\zeta$-potential of the liposomes remained almost constant upon successive addition of $\mathrm{PSO}_{4}$. There was no evidence for the formation of a micelle or a bicelle ${ }^{30}$ in the system. The observed tendency in the effect of $\mathrm{PSO}_{4}$ on the $\zeta$-potential of the PC liposomes is explained as follows. The $\mathrm{PSO}_{4}$ molecules incorporated in the PC bilayers increase not only the number of charged groups in the vicinity of the bilayer interface but also the spacing between headgroups. This phenomenon reduces the number of molecules per unit area, which leads to a decrease in the surface charge density, thus resulting in a lower value of $\zeta$-potential than expected ${ }^{311}$. Moreover, $\mathrm{PSO}_{4}$ gives rise to a corrugation along the membrane surface. In the case of DPPC, such corrugation is repeated at regular intervals in a well-defined bilayer ripple phase $\left(\mathrm{P}_{\beta}\right)^{3)}$. Though such a well-defined phase has not so far been reported in the case of DOPC, it is expectable that in this case too, $\mathrm{PSO}_{4}$ introduces such irregularities in membrane surface. The corrugated charged surfaces act as an obstacle for the free movement of the solvent layer and restricts its mobility ${ }^{32,33)}$, resulting in a reduced $\zeta$-potential. Of course, the contribution from the lowered degree of dissociation of $\mathrm{PSO}_{4}$ caused by its increased surface density also cannot be neglected in this context.

\subsection{Phase transition}

DSC heating endotherms of the DPPC-PSO ${ }_{4}$ and DOPC-

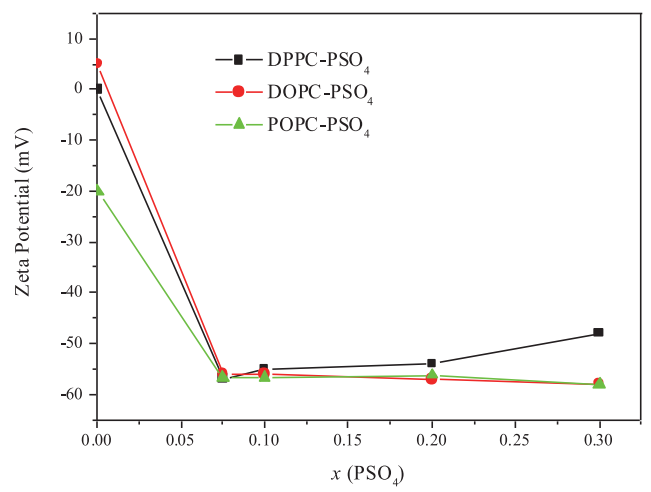

Fig. 3 Variation of hydrodynamic diameter $\left(D_{H}\right)$ (left) and $\zeta$-potential(right) of the DPPC and DOPC liposomes as a function of the mole fraction of $\mathrm{PSO}_{4}(x)$. 
$\mathrm{PSO}_{4}$ liposomes are shown in Fig. 4. In the case of the POPC-systems, the phase transition peaks were masked by the intense ice-melting peak located near $0^{\circ} \mathrm{C}$ because of the closeness of the two, and has not been shown here. The main transition temperatures $\left(\mathrm{T}_{\mathrm{m}}\right)$ of $-17^{\circ} \mathrm{C}$ and $42.1^{\circ} \mathrm{C}$, respectively for DOPC and DPPC are consistent with the previously reported values ${ }^{3,14,34,35)}$. The large difference in $\mathrm{T}_{\mathrm{m}}$ of the two PCs is due to the loose packing of the cis- hydrocarbon chains in DOPC bilayers compared to the all-trans chains in DPPC. The pre-transition peak of DPPC exists as a small, diffused peak located at $37.3^{\circ} \mathrm{C}$. The pre-transition temperature $\left(\mathrm{T}_{\mathrm{p}}\right)$ is a characteristic of the bilayers made of saturated PCs. It represents the transition from the gel $\left(\mathrm{L}_{\beta}{ }^{\prime}\right)$ phase to the rippled $\left(\mathrm{P}_{\beta}{ }^{\prime}\right)$ phase $^{3,14,35,36)}$. In the case of DPPC, this phase forms when the concentration of water is above $18 \mathrm{wt}{ }^{37)}$. However, the formation of the $\mathrm{P}_{\beta}^{\prime}$ in a similar way has not been reported in the cases of the unsaturated PCs such as DOPC.

The effect of the addition of $\mathrm{PSO}_{4}$ on these two types of bilayers, however, is comparable. In both cases, $\mathrm{PSO}_{4}$ lowered the $\mathrm{T}_{\mathrm{m}}$ peak maxima by $\sim 2^{\circ} \mathrm{C}$ per 0.1 rise in $x$. It significantly broadened the DSC peaks indicating a lowered cooperativity of phase transition. The broadening of the thermograms upon addition of $\mathrm{PSO}_{4}$ up to $x=0.2$ in the gel phase regime is associated with the partial fluidization of the membrane. 0.3 Mole fractions of $\mathrm{PSO}_{4}$ abolished the main phase transition peak $\left(\mathrm{T}_{\mathrm{m}}\right)$ of DPPC but not that of DOPC, indicating a weaker influence in the later bilayer at low temperatures. Fluidization of the membrane upon addition of a steroid has been reported earlier in the cases of other PCs too ${ }^{3,12,13,38)}$.

\subsection{Membrane fluidity}

Figure 5 shows the DPH fluorescence anisotropy $\left(r_{d p h}\right)$ data for the liposomes consisting of DPPC, DOPC, and POPC. As shown in this figure, the room-temperature fluorescence anisotropy of DPPC decreases with increasing $x$ indicating that the membrane fluidity increases. The increase in fluidity of the hydrocarbon region of a membrane

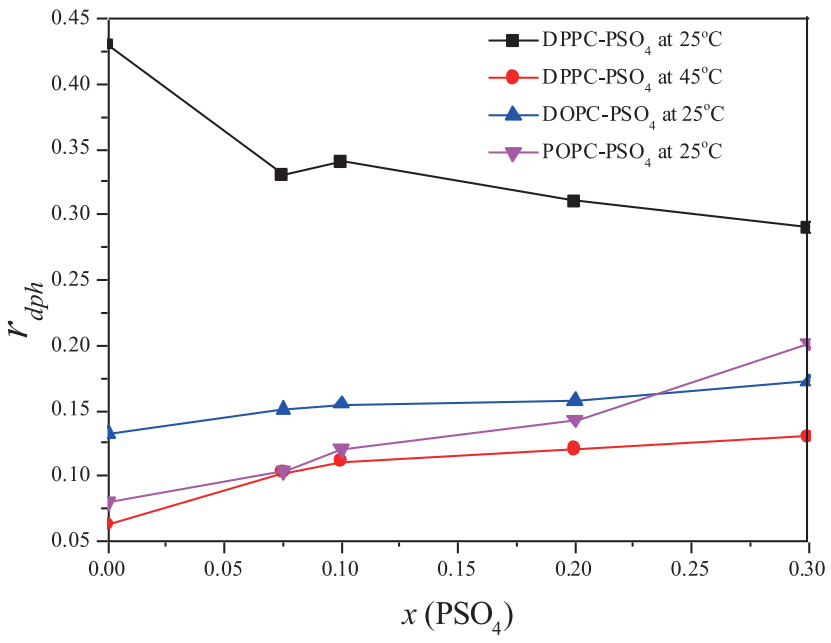

Fig. 5 DPH fluorescence anisotropy $\left(r_{d p h}\right)$ for DPPC-PSO DOPC-PSO ${ }_{4}$ and $\mathrm{POPC}-\mathrm{PSO}_{4}$ liposomes at $25^{\circ} \mathrm{C}$ and $45^{\circ} \mathrm{C}$. For DPPC in its gel phase, $r_{d p h}$ decreases with increasing $x$ indicating that $\mathrm{PSO}_{4}$ fluidizes such membrane. Slight rigidification of the $\mathrm{L}_{\alpha}$ phase membranes is observed in both cases.

in its gel phase occurs due to the disordering effect of $\mathrm{PSO}_{4}$ on the hydrocarbon chain of the lipid molecules ${ }^{3,19)}$. At $45^{\circ} \mathrm{C}$, on the other hand, the $r_{d p h}$ increases with increasing $x$ indicating an ordering effect of $\mathrm{PSO}_{4}$ in the membrane in its liquid crystalline phase. Similar ordering effect of $\mathrm{PSO}_{4}$ was observed with DOPC and POPC at room temperature for the same reason as the ordering of the DPPC membrane in the liquid crystalline $\left(\mathrm{L}_{\alpha}\right)$ phase. According to the previous reports, the ordering effect of the membrane steroids arises from their unique structural characteristics and the tendency of the phospholipid molecules to occupy fixed positions around the steroid molecules ${ }^{39,40)}$. Our previous studies ${ }^{9,38}$ have revealed that $\mathrm{PSO}_{4}$, like cholesterol and other phytosterols ${ }^{15,35}$, has both fluidizing and rigidifying effects on the PC membranes depending on whether the membrane is in a gel or a liquid crystalline phase. However, it has a higher fluidizing effect compared to other sterols ${ }^{3,41}$,
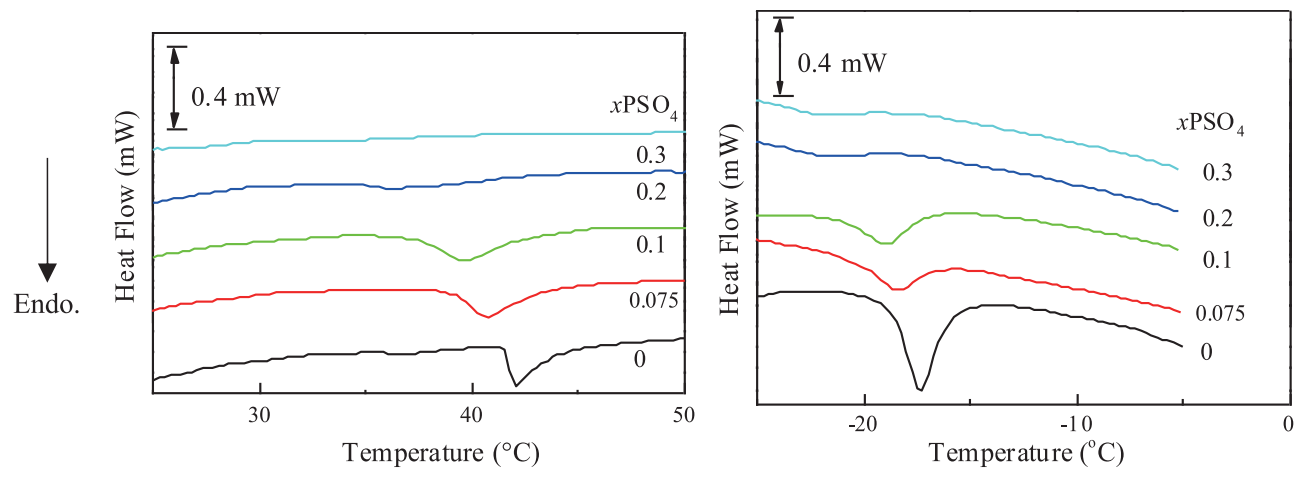

Fig. 4 DSC heating endotherms for DPPC (left) and DOPC (right) liposomes containing varying mole fractions $(x)$ of $\mathrm{PSO}_{4}$. Lipid concentration $=10 \mathrm{mM}$. 
mainly owing to its bulky sulfate moiety.

\subsection{Trapping efficiency}

Glucose trapping efficiencies (TE) of DPPC and DOPC liposomes containing 0 to 0.5 mole fraction of $\mathrm{PSO}_{4}$ were evaluated. The effect of $\mathrm{PSO}_{4}$ was compared with the effect of $\beta$-sitosterol ( $\mathrm{POH})$. To evaluate the effect, if any, of the isomeric composition and the origin of products in the role played by steroids, we used two different samples of $\mathrm{POH}$, obtained respectively from LVMC Inc. and Sigma. The steroid sample obtained from LVMC Inc. was in the form of a mixture of $\beta$-sitosterol $(\mathrm{POH})$ and $\mathrm{PSO}_{4}$. We isolated the two components from the mixture and used separately for glucose trapping efficiency experiments. The $\beta$-sitosterol sample obtained from Sigma, on the other hand, was used as is. The trapping efficiencies were determined with the help of UV-Visible spectrophotometry of the dialyzed liposome samples hydrated with glucose solution.

UV-Vis Spectrophotometry

Absorbances of the solutions treated with GOD assay (Fig. S3, Supplementary Information) were measured using a UV-visible spectrophotometer at $\lambda_{\max }=505 \mathrm{~nm}$. Concentration of the trapped glucose was determined in each solution against a calibration curve plotted using standard glucose solutions provided by the supplier of the GOD assay. The values of the trapping efficiencies (TE) of the DPPC and DOPC liposomes with and without steroids are given in Table 1. The TE for pure DPPC liposomes equal to $1.8 \%$, is comparable to that obtained by Otake $e t$ $a{ }^{42)}$.

The data on Table 1 show that $\mathrm{PSO}_{4}$ enhances the TE of DPPC liposomes much more significantly than POH. For example, $\mathrm{POH}$ at a concentration of $x=0.1$ increases the TE of pure DPPC liposome from $1.8 \%$ to $\sim 3.5 \%$ while the same amount of $\mathrm{PSO}_{4}$ enhances it to $\sim 12 \%$. The liposome of pure DOPC has a trapping efficiency (TE) of approx. $2.5 \%$ which is slightly higher than the TE of DPPC (1.8). The slightly higher TE of the DOPC liposome can be attributed to its longer hydrocarbon chains (by two carbon units)compared to DPPC and the presence of two double bonds ${ }^{43,44)}$. Upon addition of 0.05 mole fraction of $\mathrm{PSO}_{4}$, the TE increases to $9.3 \%$. It shows that $\mathrm{PSO}_{4}$ enhances TE of liposomes regardless of whether the phospholipid is saturated or unsaturated. A notable difference between the DPPC and DOPC liposomes, however, is that in the case of DPPC, the trapping efficiency first increases with the increasing concentration of $\mathrm{PSO}_{4}$ and then decreases, while in the case of DOPC, once $\mathrm{PSO}_{4}$ is incorporated, the TE remains nearly same regardless of the concentration of $\mathrm{PSO}_{4}$. This result can be explained on the basis of the variation of the size and the membrane properties of the vesicles caused by the addition of $\mathrm{PSO}_{4}$.

As can be seen in Table 1, the size of pure DPPC liposomes is over three times bigger than the size of the DOPC liposomes. It's because of the fact that the MLV of DPPC, which is in the gel phase, possesses a larger number of bilayers compared to the DOPC MLV. Thus, it can be said that the TE is higher for the system consisting of a larger number of vesicles with fewer bilayers than the system consisting of a smaller number of vesicles with a larger number of bilayers. The TE of the DOPC liposomes containing $\mathrm{PSO}_{4}$ are uniform throughout the whole composition range. This can be attributed to the uniform sizes of the vesicles and a smaller effect of $\mathrm{PSO}_{4}$ on the membrane fluidity $\left(\mathrm{PSO}_{4}\right.$ slightly rigidifies the DOPC MLVs which are initially in a liquid crystalline phase). Phases are also uniformly the $\mathrm{L}_{0}$ and $\mathrm{L}_{\alpha}$ (both fluid phases) phases in the case of the DOPC-PSO ${ }_{4}$ compared to the DPPC-PSO ${ }_{4}$ liposomes

Table 1 Trapping of glucose by the DPPC-POH and DPPC-PSO 4 liposomes.

\begin{tabular}{|c|c|c|c|c|c|c|c|c|}
\hline \multirow[b]{3}{*}{$x$} & \multicolumn{8}{|c|}{ Trapping efficiency $(\mathrm{TE})(\%)=\frac{\text { Amount of glucose in liposomes }}{\text { Glucose of the whole system }}$} \\
\hline & \multicolumn{4}{|c|}{ Size $(\mathrm{nm})^{*}$} & \multicolumn{4}{|c|}{ Trapping efficiency (\%) } \\
\hline & DPPC-PSO ${ }_{4}$ & $\begin{array}{l}\text { DPPC-POH } \\
\text { Sigma }\end{array}$ & $\begin{array}{l}\text { DPPC-POH } \\
\text { LVMC }\end{array}$ & $\begin{array}{l}\mathrm{DOPC}- \\
\mathrm{PSO}_{4}\end{array}$ & $\mathrm{DPPC}_{-} \mathrm{PSO}_{4}$ & $\begin{array}{l}\text { DPPC-POH } \\
\text { Sigma }\end{array}$ & $\begin{array}{l}\text { DPPC-POH } \\
\text { LVMC }\end{array}$ & $\begin{array}{c}\mathrm{DOPC}- \\
\mathrm{PSO}_{4}\end{array}$ \\
\hline 0 & 6619 & 7133 & 5694 & 2168 & 1.8 & 1.8 & 1.8 & 2.5 \\
\hline 0.05 & 836 & 4236 & 3148 & 654 & 8.1 & 1.7 & 1.9 & 9.3 \\
\hline 0.075 & 888 & 4260 & 3468 & 664 & 11.7 & 2.8 & 2.0 & 7.5 \\
\hline 0.1 & 601 & 2823 & 3100 & 648 & 11.7 & 3.5 & 3.0 & 7.1 \\
\hline 0.15 & 157 & 3847 & 2651 & 665 & 10.6 & 2.3 & 1.8 & 7.7 \\
\hline 0.2 & 231 & 2690 & 2938 & 705 & 7.2 & 2.5 & 2.3 & 7.7 \\
\hline 0.3 & 329 & 2619 & 2545 & 631 & 4.7 & 2.0 & 2.6 & 9.0 \\
\hline 0.4 & 728 & 3468 & 2687 & 661 & 2.8 & 3.5 & 4.6 & 7.3 \\
\hline 0.5 & 6641 & 3100 & 2854 & 640 & 5.5 & 4.7 & 9.0 & 7.7 \\
\hline
\end{tabular}

*Note: The sizes plotted in Table 1 correspond to the $10 \mathrm{mM}$ liposomal dispersions prepared in $0.027 \mathrm{M}$ glucose solution. 


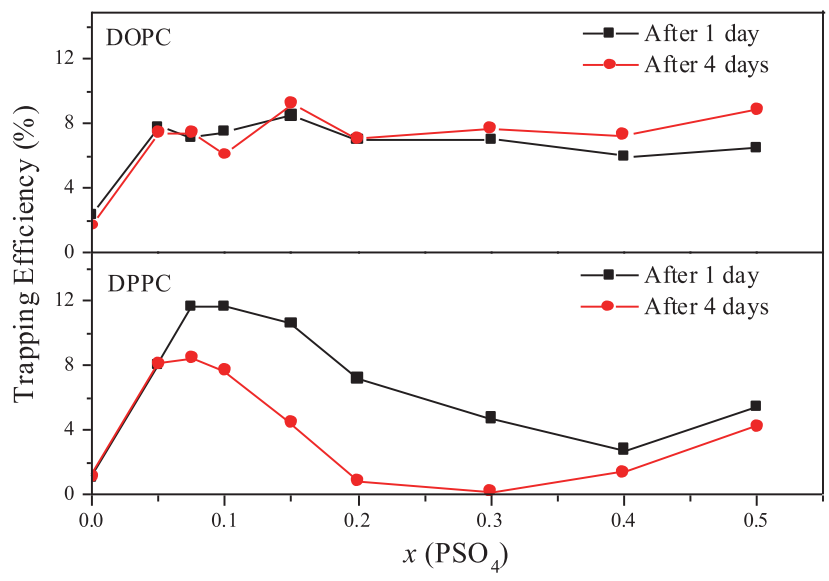

Fig. 6 Trapping efficiencies of the DOPC-PSO ${ }_{4}$ liposomes (top) and $\mathrm{DPPC}-\mathrm{PSO}_{4}$ liposomes (bottom) as a function of $x$ after 1 day and 4 days of sample preparation.

which exhibit a diversity of phases ${ }^{3,9)}$.

The larger aqueous volume in the $\mathrm{PSO}_{4}$-containing liposomes $^{9,38}$ plays a major role in their high trapping efficiencies. The other contributing factor is the formation of a large number of smaller-sized liposomes with less number of bilayers in place of the originally present large-sized multilamellar liposomes. Though increase in TE to some extent by sterols has been reported previously ${ }^{45)}$, so far there is no report of another steroid as effective as $\mathrm{PSO}_{4}$. In the case of $\mathrm{DPPC}-\mathrm{PSO}_{4}$ liposomes, the highest TE was found for liposomes with $x$ ranging from 0.075 to 0.15 lipid composition in which, according to our previous findings ${ }^{3)}$, the ripple phase $\left(\mathrm{P}_{\beta}\right)$ is predominant. The rippled structure in the interface, combined with the loose headgroup binding caused by $\mathrm{PSO}_{4}$, results in an enhanced trapping of glucose. For $x>0.2$, the predominant phase is the liquid ordered phase, which being a fluid phase, is leakier and retains a smaller amount of glucose.

\subsection{Leakage of glucose}

The DPPC and DOPC liposomes exhibited significant differences in their behavior regarding leakage of glucose as a function of storage period. As shown in Fig. 6, there is no significant leakage of glucose from the DOPC liposomes upon storage for over four days. This tendency is different from the corresponding DPPC systems in which the glucose TE decreased significantly upon storage. This can be attributed to the fact that in DOPC liposomes which undergo the $\mathrm{L}_{\alpha} \rightarrow \mathrm{L}_{\mathrm{o}}$ (fluid to fluid) transition upon addition of $\mathrm{PSO}_{4}$, there is much less structural perturbation than DPPC which undergoes a gel $\rightarrow$ fluid phase transition. Higher the structural perturbation, higher can be the number of defects in the bilayer leading to a higher leakiness.

\section{Conclusions}

Effects of $\beta$-sitosteryl sulfate $\left(\mathrm{PSO}_{4}\right)$ on the liposomal properties of saturated, mono-unsaturated, and di-unsaturated phosphatidylcholines were studied comparatively. $\mathrm{PSO}_{4}$ reduced the sizes of the liposomal particles of each of DPPC (saturated), POPC (monounsaturated), and DOPC (di-unsaturated). However, the decrease in size of DPPC was the most significant. It also enhanced the $\zeta$-potentials of both saturated and unsaturated liposomes, thus ensuring an enhanced dispersibility compared to the liposomes obtained from pure PCs. The negative surface charge induced by $\mathrm{PSO}_{4}$ is responsible for the observed effects on both the size and $\zeta$-potential. In both cases, $\mathrm{PSO}_{4}$ rigidified the membranes in their fluid phase. Fluidization of the gel phase DPPC membrane was also observed. It also significantly enhanced glucose trapping efficiencies of both DOPC and DPPC liposomes. However, the two showed significant differences in the trend of variation of $\mathrm{TE}$ with $\mathrm{PSO}_{4}$ concentration. In the case of DOPC, when a small amount of $\mathrm{PSO}_{4}$ is added, the TE increases once and then remains unaffected by further addition. But in the case of DPPC, TE first rises and then gradually drops with increasing $\mathrm{PSO}_{4}$ concentrations. This difference is attributed to the uniformity in phase structure of DOPC regardless of the concentration of $\mathrm{PSO}_{4}$ compared to DPPC which undergoes various phase changes. The TE also depends on the particle sizes of liposomes, and hence, the diversity observed in the sizes of DPPC liposomes upon addition of $\mathrm{PSO}_{4}$ is also reflected in its TE. POH, due to its lack of capacity for enhancing membrane hydration, has far less effect than $\mathrm{PSO}_{4}$. The ability of $\mathrm{PSO}_{4}$ for reducing liposomal sizes, and enhancing dispersibility, stability, and trapping efficiencies is useful for formulating cosmetics and drug delivery systems.

\section{Supporting Information}

This material is available free of charge via the Internet at doi: $10.5650 /$ jos.ess 21035

\section{References}

1) Gu, R.X.; Baoukina, S.; Peter Tieleman, D. Phase separation in atomistic simulations of model membranes. $J$. Am. Chem. Soc. 142, 2844-2856 (2020).

2) Perczyk, P.; Wójcik, A.; Hachlica, N.; Wydro, P.; Broniatowski, M. The composition of phospholipid model bacterial membranes determines their endurance to secretory phospholipase A2 attack - The role of cardiolipin. Biochim. Biophys. Acta 1862, 183239 (2020).

3) Kafle, A.; Misono, T.; Bhadani, A.; Sakai, K.; Kaise, C.; 
Kaneko, T.; Sakai, H. Effects of sodium $\beta$-sitosteryl sulfate on the phase behavior of dipalmitoylphosphatidylcholine. Colloids Surf. B 161, 59-66 (2018).

4) Vemuri, S.; Rhodes, C.T. Preparation and characterization of liposomes as therapeutic delivery systems: a review. Pharm. Acta Helv. 70, 95-111 (1995).

5) Pagano, R.E.; Weinstein, J.N. Interactions of liposomes with mammalian cells. Annu. Rev. Biophys. Bioeng. 7, 435-468(1978).

6) Sierra, M.B.; Pedroni, V.I.; Buffo, F.E.; Disalvo, E.A.; Morini, M.A. The use of zeta potential as a tool to study phase transitions in binary phosphatidylcholines mixtures. Colloids Surf. B 142, 199-206 (2016).

7) Wiacek, A.E. Influence of dipalmitoylphosphatidylcholine (or dioleoylphosphatidylcholine) and phospholipase A2 enzyme on the properties of emulsions. $J$. Colloid Interface Sci. 373, 75-83 (2012).

8) Lou, J.; Best, M.D. A General approach to enzyme-responsive liposomes. Chem. - A Eur. J. 26, 8597-8607 (2020).

9) Kafle, A.; Akamatsu, M.; Bhadani, A.; Sakai, K.; Kaise, C.; Kaneko, T.; Sakai, H. Effects of $\beta$-sitosteryl sulfate on the properties of DPPC liposomes. J. Oleo Sci. 67, 1511-1519 (2018).

10) Bernsdorff, C.; Winter, R. Differential properties of the sterols cholesterol, ergosterol, beta-sitosterol, trans7-dehydrocholesterol, stigmasterol and lanosterol on DPPC bilayer order. J. Phys. Chem. B 107, 1065810664 (2003).

11) St. Clair, J.W.; London, E. Effect of sterol structure on ordered membrane domain (raft) stability in symmetric and asymmetric vesicles. Biochim. Biophys. Acta 1861, 1112-1122 (2019).

12) Benesch, M.G.K.; Lewis, R.N.A.H.; McElhaney, R.N. A calorimetric and spectroscopic comparison of the effects of cholesterol and its sulfur-containing analogs thiocholesterol and cholesterol sulfate on the thermotropic phase behavior and organization of dipalmitoylphosphatidylcholine bilayer membranes. Biochim. Biophys. Acta 1858, 168-180 (2016).

13) Schofield, M.; Jenski, L.J.; Dumaual, A.C.; Stillwell, W. Cholesterol versus cholesterol sulfate: effects on properties of phospholipid bilayers containing docosahexaenoic acid. Chem. Phys. Lipids 95, 23-36 (1998).

14) Wu, R.G.; Chen, L.; Yu, Z.; Quinn, P.J. Phase diagram of stigmasterol-dipalmitoylphosphatidylcholine mixtures dispersed in excess water. Biochim. Biophys. Acta 1758, 764-771 (2006).

15) Chen, L.; Yu, Z.; Quinn, P.J. The partition of cholesterol between ordered and fluid bilayers of phosphatidylcholine: A synchrotron X-ray diffraction study. Biochim. Biophys. Acta 1768, 2873-2881 (2007).

16) Mannock, D.A.; Benesch, M.G.K.; Lewis, R.N.A.H.; McElhaney, R.N. A comparative calorimetric and spec- troscopic study of the effects of cholesterol and of the plant sterols $\beta$-sitosterol and stigmasterol on the thermotropic phase behavior and organization of dipalmitoylphosphatidylcholine bilayer membranes. Biochim. Biophys. Acta 1848, 1629-1638(2015).

17) Hussain, A.; Singh, S.; Sharma, D.; Webster, T.J.; Shafaat, K.; Faruk, A. Elastic liposomes as novel carriers: Recent advances in drug delivery. Int. J. Nanomedicine. 12, 5087-5108(2017).

18) Brewer, M.S. Current status of bovine spongiform encephalopathy-A review. J. Muscle Foods 10, 97-117 (1999).

19) Kafle, A.; Misono, T.; Bhadani, A.; Akamatsu, M.; Sakai, K.; Kaise, C.; Kaneko, T.; Sakai, H. Effects of $\beta$-sitosteryl sulfate on the phase behavior and hydration properties of distearoylphosphatidylcholine: A comparison with dipalmitoylphosphatidylcholine. $J$. Oleo Sci. 67, 433-443(2018).

20) Kafle, A.; Akamatsu, M.; Bhadani, A.; Sakai, K.; Kaise, C.; Kaneko, T.; Sakai, H. Phase behavior of the bilayers containing hydrogenated soy lecithin and $\beta$-sitosteryl sulfate. Langmuir 36, 6025-6032 (2020).

21) Akbarzadeh, A.; Rezaei-Sadabady, R.; Davaran, S.; Joo, S.W.; Zarghami, N.; Hanifehpour, Y.; Samiei, M.; Kouhi, M.; Nejati-Koshki, K. Liposome: Classification, preparation, and applications. Nanoscale Res. Lett. 8, 102110 (2013).

22) Imura, T.; Sakai, H.; Yamauchi, H.; Kaise, C.; Kozawa, K.; Yokoyama, S.; Abe, M. Preparation of liposomes containing Ceramide 3 and their membrane characteristics. Colloids Surf. B 20, 1-8(2001).

23) Lentz, B.R.; Moore, B.M.; Barrow, D.A. Light-scattering effects in the measurement of membrane microviscosity with diphenylhexatriene. Biophys. J. 25, 489-494 (1979).

24) Atkinson, D.; Hauser, H.; Shipley, G.G.; Stubbs, J.M. Structure and morphology of phosphatidylserine dispersions. Biochim. Biophys. Acta 339, 10-29 (1974).

25) Bergmeier, M.; Gradzielski, M.; Hoffmann, H.; Mortensen, K. Behavior of ionically charged lamellar systems under the influence of a shear field. J. Phys. Chem. B 103, 1605-1617 (1999).

26) Claessens, M.M.A.E.; Van Oort, B.F.; Leermakers, F.A.M.; Hoekstra, F.A.; Stuart, M.A.C. Charged lipid vesicles: Effects of salts on bending rigidity, stability, and size. Biophys. J. 87, 3882-3893(2004).

27) Kwok, R.; Evans, E. Thermoelasticity of large lecithin bilayer vesicles. Biophys. J. 35, 637-652 (1981).

28) Choi, S.-E.; Greben, K.; Wördenweber, R.; Offenhäusser, A. Positively charged supported lipid bilayer formation on gold surfaces for neuronal cell culture. Biointerphases 11, 021003 (2016).

29) Chibowski, E.; Szczes, A. Zeta potential and surface charge of DPPC and DOPC liposomes in the presence 
of PLC enzyme. Adsorption 22, 755-765(2016).

30) Watanabe, Y.; Aramaki, K.; Kadomatsu, Y.; Tanaka, K.; Konno, Y. Preparation of bicelles using the semi-spontaneous method. Chem. Lett. 45, 558-560(2016).

31) Matos, C.; De Castro, B.; Gameiro, P.; Lima, J.L.F.C.; Reis, S. Zeta-potential measurements as a tool to quantify the effect of charged drugs on the surface potential of egg phosphatidylcholine liposomes. Langmuir 20, 369-377 (2004).

32) Joly, L.; Ybert, C.; Trizac, E.; Bocquet, L. Hydrodynamics within the electric double layer on slipping surfaces. Phys. Rev. Lett. 93, 1-4(2004).

33) Kim, Y.W.; Netz, R.R. Electro-osmosis at inhomogeneous charged surfaces: Hydrodynamic versus electric friction. J. Chem. Phys. 124, 114709 (2006).

34) Bulavin, L.A.; Soloviov, D.V.; Gordeliy, V.I.; Svechnikova, O.S.; Krasnikova, A.O.; Kasian, N.A.; Vashchenko, O.V.; Lisetski, L.N. Lyotropic model membrane structures of hydrated DPPC: DSC and small-angle X-ray scattering studies of phase transitions in the presence of membranotropic agents. Phase Transitions 88, 582-592 (2015).

35) Kamal, M.A.; Raghunathan, V.A. Phase behavior of phospholipid-phytosterol membranes. Soft Matter 8, 8952-8958(2012).

36) Cunningham, B.A.; Brown, A.-D.; Wolfe, D.H.; Williams, W.P.; Brain, A. Ripple phase formation in phosphatidylcholine: Effect of acyl chain relative length, position, and unsaturation. Phys. Rev. E 58, 36623672 (1998).

37) Kodama, M.; Kuwabara, M.; Seki, S. Successive phasetransition phenomena and phase diagram of the phosphatidylcholine-water system as revealed by differential scanning calorimetry. Biochim. Biophys. Acta
689, 567-570 (1982).

38) Kafle, A.; Misono, T.; Bhadani, A.; Akamatsu, M.; Sakai, K.; Kaise, C.; Kaneko, T.; Sakai, H. Effects of $\beta$-sitosteryl sulfate on the hydration behavior of dipalmitoylphosphatidylcholine. J. Oleo Sci. 67, 763-771 (2018).

39) Martinez-Seara, H.; Rog, T.; Karttunen, M.; Vattulainen, Ii.; Reigada, R. Cholesterol induces specific spatial and orientational order in cholesterol / phospholipid membranes. PLoS One 5, 1-11(2010).

40) Czub, J.; Baginski, M. Comparative molecular dynamics study of lipid membranes containing cholesterol and ergosterol. Biophys. J. 90, 2368-2382(2006).

41) Grimellec, C.L.; Daigneault, A.; Bleau, G.; Roberts, K.D. Cholesteryl sulfate-phosphatidylcholine interactions. Lipids 19, 474-477 (1984).

42) Otake, K.; Imura, T.; Sakai, H.; Abe, M. Development of a new preparation method of liposomes using supercritical carbon dioxide. Langmuir 17, 3898-3901 (2001).

43) Otake, K.; Shimomura, T.; Goto, T.; Imura, T.; Furuya, T.; Yoda, S.; Takebayashi, Y.; Sakai, H.; Abe, M. Preparation of liposomes using an improved supercritical reverse phase evaporation method. Langmuir 22, 25432550 (2006).

44) Sakai, H.; Gotoh, T.; Imura, T.; Sakai, K.; Otake, K.; Abe, M. Preparation and properties of liposomes composed of various phospholipids with different hydrophobic chains using a supercritical reverse phase evaporation method. J. Oleo Sci. 57, 613-621 (2008).

45) Pick, U. Liposomes with a large trapping capacity prepared by freezing and thawing of sonicated phospholipid mixtures. Arch. Biochem. Biophys. 212, 186194 (1981). 